University of Nebraska - Lincoln

DigitalCommons@University of Nebraska - Lincoln

Faculty Publications, Department of Psychology

Psychology, Department of

March 2003

Impact of Nicotine Withdrawal on Novelty Reward and Related Behaviors

Joyce Besheer

University of Nebraska-Lincoln

Rick A. Bevins

University of Nebraska-Lincoln, rbevins1@unl.edu

Follow this and additional works at: https://digitalcommons.unl.edu/psychfacpub

Part of the Psychiatry and Psychology Commons

Besheer, Joyce and Bevins, Rick A., "Impact of Nicotine Withdrawal on Novelty Reward and Related Behaviors" (2003). Faculty Publications, Department of Psychology. 184.

https://digitalcommons.unl.edu/psychfacpub/184

This Article is brought to you for free and open access by the Psychology, Department of at DigitalCommons@University of Nebraska - Lincoln. It has been accepted for inclusion in Faculty Publications, Department of Psychology by an authorized administrator of DigitalCommons@University of Nebraska - Lincoln. 
Published in Behavioral Neuroscience, Vol. 117, No. 2 (2003), pp. 327-340. Copyright 2003 by the American Psychological Association, Inc. "This article may not exactly replicate the final version published in the APA journal. It is not the copy of record." Used by permission. http://www.apa.org/journals/bne/

\title{
Impact of Nicotine Withdrawal on Novelty Reward and Related Behaviors
}

\author{
Joyce Besheer and Rick A. Bevins \\ University of Nebraska-Lincoln
}

\begin{abstract}
The authors tested the decreased reward function hypothesis of nicotine withdrawal using a novel-object place conditioning task. A conditioned place preference was evident in controls and in rats that had experienced 4 nicotine withdrawal days, but not in rats that had experienced 1-3 withdrawal days. This implies that the rewarding properties of interacting with novel objects were not readily associated with the environment in which they were paired. Follow-up experiments eliminated other explanations based on withdrawal-induced failures to process object or environment information. Also, expression of conditioning was not affected, indicating that withdrawal likely altered acquisition. Further investigation into the neurochemical and behavioral changes that accompany nicotine withdrawal will lead to a better understanding of the withdrawal syndrome.
\end{abstract}

Compulsive tobacco use continues to be a national public health problem. About 46 million adults in the United States are current smokers (Centers for Disease Control and Prevention [CDC], 2001). Surprisingly, approximately $70 \%$ of the adult smokers in the United States report a desire to quit. However, out of these smokers, only about $3 \%$ succeed and maintain cessation (CDC, 1994). A likely contributing factor to the high relapse rate, especially during early cessation, is the occurrence of withdrawal symptoms experienced after the cessation of chronic tobacco intake (Killen, Fortmann, Newman, \& Varady, 1991). These withdrawal symptoms include somatic disturbances such as sweating, dizziness, insomnia, and stomachache, as well as affective symptoms such as depressed mood, irritability, anxiety, and craving for tobacco products (Hughes et al., 1984; Hughes, Gust, Skoog, Keenan, \& Fenwick, 1991; Shiffman et al., 2000; West \& Hajek, 1996). A common reason why smokers relapse after a brief period of cessation is to alleviate these withdrawal symptoms. Given that the affective components of nicotine withdrawal are likely important contributing factors to cravings and relapse (Epping-Jordan, Watkins, Koob, \& Markou, 1998; Watkins, Stinus, Koob, \& Markou, 2000), a detailed investigation of the impact of withdrawal in

Joyce Besheer and Rick A. Bevins, Department of Psychology, University of Nebraska-Lincoln. This research was part of a dissertation submitted by Joyce Besheer to the University of Nebraska-Lincoln in partial fulfillment of $\mathrm{PhD}$ requirements. This work was supported by funds from Individual National Research Service Award DA06092 to Joyce Besheer and National Institute on Drug Abuse Grant DA11893 to Rick A. Bevins. We thank Daniel Leger, John Flowers, Eric Fung, and Matthew Palmatier for their thoughtful comments on an earlier version of this article. We are grateful to Dawn Metschke for conducting the independent observations used to assess interobserver reliability. Correspondence concerning this article should be addressed to Joyce Besheer, who is now at the Bowles Center for Alcohol Studies, University of North Carolina at Chapel Hill, CB \#7178, Thurston Bowles Building, Chapel Hill, North Carolina 27599-7178 or to Rick A. Bevins, Department of Psychology, University of Nebraska-Lincoln, Lincoln, Nebraska 68588-0308. Email: jbesheer@med.unc.edu or rbevins1@unl.edu an animal model is essential for a complete understanding of the behavioral and biological bases of compulsive tobacco use.

What is currently known about the neural mechanisms mediating withdrawal in humans and animals can provide insight into the affective changes (e.g., depressed mood, anxiety) that accompany smoking cessation. For example, changes in the dopaminergic system have been reported in rodents during nicotine withdrawal. Hildebrand, Nomikos, Hertel, Schilström, and Svensson (1998) found that precipitation of withdrawal during chronic nicotine treatment was followed by a decrease in dopamine levels in the nucleus accumbens. This decrease was relative to a prewithdrawal baseline and reached levels below those of non-nicotine-treated controls (see also Carboni, Bortone, Giua, \& Di Chiara, 2000; Fung, Schmid, Anderson, \& Lau, 1996). This change suggests that affective processes related to dopamine may be altered. That is, the mesolimbic dopamine system is involved in mediating response to reward; decreases in nucleus accumbens dopamine levels during nicotine withdrawal suggest that the rewarding impact of certain stimuli may be reduced (i.e., anhedonia). Indeed, research in rodent models provides direct support for this notion. In a report by Epping-Jordan et al. (1998), rats receiving chronic nicotine infusion were trained to respond for intracranial self-stimulation (ICSS). During nicotine withdrawal, ICSS thresholds were significantly elevated, suggesting that the rewarding properties of the electrical stimulation were decreased. A similar finding of elevated reward thresholds induced by cessation of systemic nicotine injections was reported by Bozarth, $\mathrm{Pu}-$ diak, and KuoLee (1998).

Given that withdrawal is associated with decreases in reward functioning as measured by ICSS, a tenable hypothesis is that learning about reward in other situations would be impaired. The major goal of this study was to test this hypothesis by examining whether the rewarding properties of novelty would be altered as assessed by a place conditioning procedure. The place conditioning preparation has commonly been used to assess the reward- 
ing, as well as the aversive, properties of various drugs of abuse (for reviews, see Bardo \& Bevins, 2000; Carr, Fibiger, \& Phillips, 1989; Tzschentke, 1998). In place conditioning, a distinct environment is repeatedly paired with the stimulus of interest, for example, cocaine. Rats receive equal confinement to another distinct environment without cocaine (saline). During a postconditioning test, rats are allowed free access to both environments (in the absence of cocaine). Rats tend to spend more time in the environment that was paired with cocaine. This preference for the cocaine-paired environment is taken to indicate that the rat has formed an association between the environment and the rewarding properties of the drug. As a result, in the absence of the drug (during the postconditioning test), the environment comes to elicit approach behaviors. This enhanced approach tendency is thought to provide a measure of the appetitive nature of the stimulus being tested (see Bardo \& Bevins, 2000; Carr et al., 1989).

Previous research from our laboratory has found that access to novelty (novel objects), like cocaine, can condition a place preference (Besheer, Jensen, \& Bevins, 1999; Bevins \& Bardo, 1999; Bevins et al., 2002). This work has shown involvement of the dopaminergic system in mediating novel-object place conditioning (Besheer et al., 1999). In that report, we concluded that dopamine was involved in the formation of an association between the rewarding properties of interacting with novel stimuli and the environment in which the novelty was experienced (cf. Sutton \& Beninger, 1999). If this conclusion is correct, then the dopaminergic changes that occur during nicotine withdrawal would prevent the acquisition of the environment-reward association and, hence, a novelty-conditioned place preference. An alternative account is that the rewarding properties of novel stimulation are altered during withdrawal. That is, being in a withdrawal-induced state of anhedonia reduces the rewarding impact of interacting with the novel objects. Regardless, both accounts predict that no preference for the novelty-paired environment would be observed during the postconditioning test.

The present study assessed reward functioning at various time points of nicotine withdrawal using the novel-object place conditioning procedure. We found that during the early stages of withdrawal, place conditioning was blocked. Further, alternative explanations to impairments in novelty reward were assessed. These follow-up experiments found that withdrawal did not affect the neural processing of the novel objects or environmental familiarization processes. Also, the expression of a conditioned association was not impaired by experiencing nicotine withdrawal during the postconditioning test. This investigation extends the existing literature on decreased reward functioning using the ICSS preparation $\mathrm{c}$ to a model that has been widely used to assess the rewarding properties of various stimuli, including drugs of abuse.

\section{General Method}

\section{Subjects}

Male Sprague-Dawley rats from Harlan Industries (Indianapolis, IN) were used in the present study. Rats were housed individually in plastic tubs lined with aspen wood chips. Food and water were continuously available in the home cage. The colony room was maintained on a 12hr light-dark cycle, and experiments were conducted during the light portion of the cycle.

\section{Drugs}

(-)-Nicotine hydrogen tartrate (Sigma, St. Louis, MO) was used in all experiments. Nicotine was mixed in $0.9 \%(\mathrm{wt} / \mathrm{vol})$ sterile saline and brought to a $\mathrm{pH}$ of $7.0 \pm 0.1$ with a dilute sodium hydroxide solution. All doses used in this report were calculated on the basis of, and are expressed as, the salt form of the drug. Mecamylamine hydrochloride (Research Biochemicals International/Sigma, Natick, MA) prepared at a dose of $1 \mathrm{mg} / \mathrm{kg}$ was dissolved in saline and injected subcutaneously at a volume of $1 \mathrm{ml} / \mathrm{kg}$.

\section{Osmotic Pump Preparation and Implantation}

The Alzet osmotic minipumps (Model 2ML1 ; Durect Corporation, Cupertino, CA) were filled with the appropriate nicotine dose and primed in sterile saline $\left(37^{\circ} \mathrm{C}\right)$ for at least $4 \mathrm{hr}$ prior to implantation. Rats were anesthetized with an intraperitoneal injection of ketamine hydrochloride $(100 \mathrm{mg} / \mathrm{ml})$ at a volume of $0.7 \mathrm{mg} / \mathrm{ml}$ and xylazine hydrochloride $(20$ $\mathrm{mg} / \mathrm{ml}$ ) solution at a volume of $0.4 \mathrm{mg} / \mathrm{ml}$ (both from Midwest Veterinary Supply, Burnsville, MN). A midscapular incision was made, and the pumps were implanted subcutaneously between the scapulae, parallel to the spine. The incision was closed with wound clips. In all the experiments, the control group received the same surgical procedures as the nicotine group. In Experiments 2 and 3, rats in the control group were implanted with previously used pumps that had been emptied, cleaned thoroughly with alcohol, and wrapped in Parafilm wax paper (see Panagis, Hildebrand, Svensson, \& Nomikos, 2000). In Experiments 1B, 4, and 5 , rats in the control group were implanted with a sealed bulb from a polyethylene pipet (VWR brand, $8 \mathrm{ml}$; VWR, West Chester, PA) that was cleaned in alcohol and was similar in size and shape to the osmotic pump (see Carboni et al., 2000).

\section{Experiment 1A: Spontaneous Nicotine Withdrawal}

Before evaluating the effects of nicotine withdrawal in the place conditioning preparation, we first had to demonstrate the presence of an overt somatic withdrawal reaction as reported by others. In the majority of studies examining nicotine withdrawal, nicotine was delivered by an osmotic pump (Epping-Jordan et al., 1998; Hildebrand et al., 1998; Hildebrand, Panagis, Svensson, \& Nomikos, 1999; Malin et al., 1992; Panagis, Hildebrand, Svensson, \& Nomikos, 2000). The osmotic pump allows for the continuous delivery of nicotine at a controlled rate for a specified amount of time. An advantage of this delivery protocol is that nicotine levels in the bloodstream remain fairly constant, much like those of human chronic smokers. Discontinuing the delivery of nicotine by removal of the pump or interfering with nicotine's effects on the central nervous system induces a withdrawal reaction.

Accordingly, this experiment sought to replicate previous work using a rodent model of nicotine withdrawal developed by Malin et al. (1992) that has become widely used (Adams \& Cicero, 1998; Epping-Jordan et al., 1998; Hildebrand et al., 1998, 1999; Panagis et al., 2000), and which implements the osmotic pump as the nicotine delivery mechanism. This model, which was adapted from a rodent model of opiate abstinence (Gianutosos, Drawbaugh, Hynes, \& Lal, 1975), relies on observing the frequency of specific somatic signs (e.g., teeth chatters, writhes, yawns) that occur spontaneously after the cessation of chronic nicotine administration by removal of the osmotic pump (Experiment 1A) or after the administration of a nicotinic acetylcholine receptor antagonist (see Experiment 1B). The frequency of these symptoms serves as an indication of the severity of the withdrawal reaction. For exam- 
ple, after 1 week of chronic nicotine delivery, removal of the osmotic pump induces a withdrawal reaction that tends to be strongest (i.e., most symptoms) during the first $48 \mathrm{hr}$ after pump removal (Epping-Jordan et al., 1998; Malin et al., 1992).

\section{Method}

Apparatus. Two clear plastic tubs $(24 \mathrm{~cm}$ long $\times 21.5 \mathrm{~cm}$ wide $\times 20$ $\mathrm{cm}$ high) served as the observation chambers. With the exception of the front wall of the chambers, which remained uncovered for videotaping of the rats, the outside walls of each chamber were lined with a white paper towel so that movement from one rat would not affect the behavior of the adjacent rat. A Plexiglas lid with drilled air holes was fastened to the top of the chambers with Velcro strips. The chambers were wiped with a diluted isopropyl alcohol solution after each rat was removed.

Procedure. Rats were placed in the observation chamber for $10 \mathrm{~min}$ on Days 1 and 2 to allow habituation to the environment. Data were not collected on these days. On Day 3, rats were returned to the chambers, and behavioral observations were made. These observations served as a baseline measure of the behaviors of interest. On Day 4, rats in the $3 \mathrm{mg} / \mathrm{kg}$ and $9 \mathrm{mg} / \mathrm{kg}$ groups ( $n=8$ per group) were implanted with osmotic pumps filled to deliver approximately 3 or $9 \mathrm{mg} / \mathrm{kg}$ of nicotine per day, respectively $(1.05$ or $3.16 \mathrm{mg} / \mathrm{kg}$ base form, respectively). As a result of weight gain during the infusion period, the amount of nicotine delivered for the $3 \mathrm{mg} /$ $\mathrm{kg}$ group ranged from $3.10 \mathrm{mg} / \mathrm{kg} 24 \mathrm{hr}$ after pump implantation and gradually declined to $2.76 \mathrm{mg} / \mathrm{kg}$ on the day of pump removal. For the $9 \mathrm{mg} /$ $\mathrm{kg}$ group, the range was $9.49-8.41 \mathrm{mg} / \mathrm{kg}$. Rats in the control group $(n=8)$ received the identical surgical procedures, but a pump was not implanted. On the day of surgery, rats weighed an average of $293 \pm 3 \mathrm{~g}$.

On Days 7 and 10, rats were placed in the observation chambers for $10 \mathrm{~min}$. These placements served to maintain the familiarity of the chambers, and behavioral observations were not made. The osmotic pumps were removed on Day 11 (after 1 week of chronic nicotine delivery); controls received sham surgery. On Days 12, 13, 14, and 15 (1, 2, 3, and 4 days after pump removal, respectively) rats were placed in the observation chambers for $10 \mathrm{~min}$, and behavioral observations were recorded. The 1st day after pump removal (i.e., Day 12) was designated as Withdrawal Day 1 (WD1), the 2nd day as WD2, and so on.

Behavioral observations and data analyses. Behavioral observations were made for the first $10 \mathrm{~min}$ of placement in the observation chamber. The frequency of behaviors in the following categories (see EppingJordan et al., 1998) were recorded: abdominal constrictions - arching of the back that was not accompanied by yawning and dragging of the abdomen (both of which can be defined as writhes), or gasps; eyeblinksrapid closing, then opening, of both eyes; facial fasciculations - bouts of chewing or teeth chattering; wet-dog shakes - full-body or head-only shakes, neither of which were associated with grooming; and miscellaneous signs - bouts of genital grooming, ptosis, or yawns. For this experiment and Experiment 1B, a second rater, who was unaware of the drug treatment received by the rats, assessed the reliability of the observations conducted by the primary rater (J. Besheer). The correlation between the total somatic signs for 18 rats was high, $r_{\mathrm{s}}=.921$.

Because of the small sample size and heterogeneity of variance, nonparametric statistics were used for all the analyses in the present report. Kruskal-Wallis tests $(H)$ were used to assess group differences on each observation session. Dunn's multiple comparison tests were used to compare each group to the control group when a significant main effect was observed. Significance level was set at $\mathrm{p} \leq .05$ (two-tailed) for all tests in this study.

\section{Results and Discussion}

Figure 1A illustrates the median of the total somatic signs observed during the baseline observation session and the 4 days after pump removal for the control and nicotine-treated groups. The total number of somatic signs did not differ
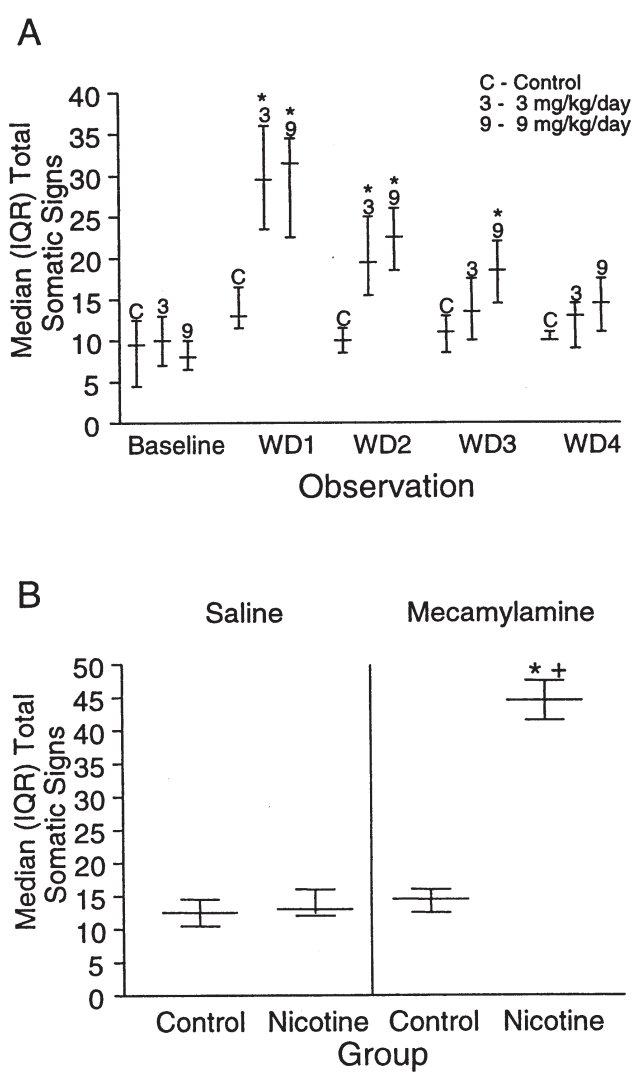

Figure 1. A: Median and interquartile range (IQR) of the total somatic signs observed during the baseline observation session and the 4 days after pump removal (withdrawal days [WD]) for each group. Asterisks indicate a significant difference from the control group $(p<.05)$. B: Median and IQR of total number of somatic signs observed after the saline and mecamylamine injections for Groups Control and Nicotine. Asterisk indicates a significant difference from the control group after the mecamylamine injection $(p<.05)$. Plus sign indicates a significant difference from the nicotine group after saline injection $(p<.05)$.

for the three groups in the initial baseline observation, $H(2)$ $=1.30, p=.52$. Group differences emerged on WD1, $H(2)=$ $11.38, p<.01$; WD2, $H(2)=13.86, p<.01$; and WD3, $H(2)$ $=9.74, p<.08$. During WD1 and WD2, both nicotine-treated groups displayed significantly more withdrawal signs than controls ( $p \mathrm{~s}<.01$ ). On WD3, only the $9 \mathrm{mg} / \mathrm{kg}$ nicotine group displayed greater withdrawal signs than the controls $(p<.01)$. There were no statistical differences by WD4, $H(2)=4.41, p$ $=.11$. The group medians (interquartile range), Kruskat-Wallis $H$ statistic, and $p$ value for each individual behavior observed during withdrawal are presented in Table 1. In sum, the data patterns observed in this experiment replicated those of previous work. Further, given the longer duration of the withdrawal reaction in the $9 \mathrm{mg} / \mathrm{kg}$ group, this dose was used throughout the remaining experiments.

\section{Experiment 1B : Mecamylamine-Precipitated Nicotine Withdrawal}

Another method commonly used to precipitate a nicotine withdrawal reaction is to inject the antagonist mecamylamine 
Table 1

Median (IQR) Frequency of Somatic Signs Measured During Spontaneous Nicotine Withdrawal

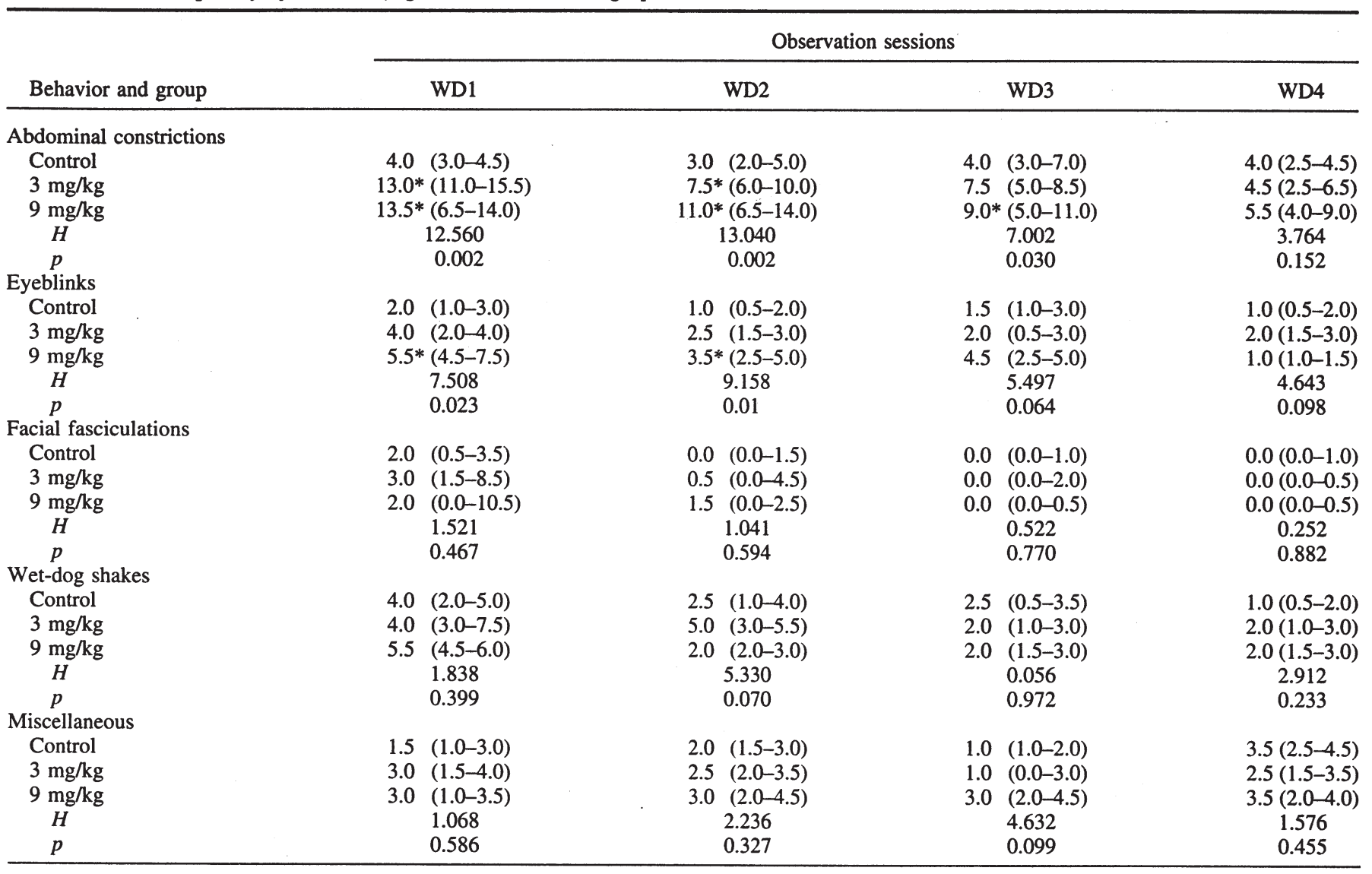

Note. $\mathrm{IQR}=$ interquartile range; $\mathrm{WD}=$ withdrawal day.

$* p<.05$ compared with controls.

(Hildebrand et al., 1998, 1999; Malin et al., 1994; Suzuki, Ise, Tsuda, Maeda, \& Misawa, 1996; Suzuki, Ise, Maeda, \& Misawa, 1999). Mecamylamine precipitates withdrawal by antagonizing both centrally and peripherally located nicotinic acetylcholine receptors (Martin, Onaivi, \& Martin, 1989), thus blocking the action of nicotine. As in spontaneous nicotine withdrawal, the nicotine-treated rats display an increase in the total number of somatic signs when compared with mecamylamine-injected controls. As in Experiment 1A, in order to use this method to precipitate nicotine withdrawal in our laboratory, we first had to replicate previous findings of a nicotine withdrawal reaction precipitated by mecamylamine. We utilized a design similar to that used by Hildebrand et al. (1998), which allows between- and within-group comparisons. A notable advantage of this design is that within-group comparisons allowed us to reduce the number of groups and thus the number of rats required for testing.

\section{Method}

Apparatus and procedure. The observation chambers were the same as those described in Experiment 1A. On the day of surgery (Day 0), the average weight of the rats was $252 \pm 3 \mathrm{~g}$. Rats were implanted with nicotine-filled osmotic pumps (nicotine group, $n=8$ ) or the sealed bulb (control group, $n=8$ ). Due to weight gain across the week of nicotine delivery, the concentration of nicotine delivered ranged from $9.90 \mathrm{mg} / \mathrm{kg}$ on Day 1 to $8.57 \mathrm{mg} / \mathrm{kg}$ on Day 7 .

On Days 5 and 6, all rats received a subcutaneous saline injection and were returned to the home cage; $15 \mathrm{~min}$ later, the rats were placed in the observation chamber for $10 \mathrm{~min}$. These 2 days served to habituate the rats to the injection protocol and the observation chambers. On Day 7, all rats were injected subcutaneously with saline $15 \mathrm{~min}$ before placement in the observation chamber; the rats remained in the observation chamber for $15 \mathrm{~min}$ before being returned to the home cage. Two hours after the initial saline injection, all rats were injected subcutaneously with mecamylamine $15 \mathrm{~min}$ before being placed in the observation chambers for $15 \mathrm{~min}$.

Behavioral observations and data analyses. As detailed in Experiment $1 \mathrm{~A}$, the frequency of abdominal constrictions, eyeblinks, facial fasciculations, wet-dog shakes, and miscellaneous signs were recorded during both 15 -min periods on Day 7. Mann-Whitney tests $(U)$ were used to evaluate group differences in the total number of somatic signs after each injection and are used throughout this report to statistically compare two groups. If a significant difference was observed in the total number of somatic signs, Mann-Whitney tests were used to evaluate differences in the frequency of the individual somatic signs. Wilcoxon signed rank tests $(T)$ were used to assess within-group differences after each injection. 


\section{Results and Discussion}

Figure 1B shows the median total number of somatic signs observed after the saline and mecamylamine injections for the control and nicotine groups. After the saline injection, the total number of symptoms in the nicotine and control groups did not differ $(U=25, p>.50)$. Thus, the continuous delivery of nicotine for 7 days did not affect the number of overt somatic signs observed. After the mecamylamine injection, the nicotine-treated rats displayed a significant increase in the total number of somatic signs $(T=36, p<.01)$. Mecamylamine alone did not increase the number of withdrawal symptoms. That is, the number of withdrawal symptoms in the control group after the saline injection and after the mecamylamine injection did not significantly differ $(T=19, p>.06)$. Further, the between-group comparison revealed that mecamylamine precipitated significantly more withdrawal signs in the nicotine-treated group than in the control group $(U=0, p<$ $.01)$. The frequency of the individual behaviors observed after each injection for both groups is illustrated in Table 2. This successful replication of the previous research that induced a withdrawal reaction by a subcutaneous injection of mecamylamine allowed us to use the $1 \mathrm{mg} / \mathrm{kg}$ mecamylamine dose in some of the following experiments to precipitate withdrawal.

\section{Experiment 2}

As discussed earlier, previous research has found that access to novel objects can condition an environmental preference (Besheer et al., 1999; Bevins \& Bardo, 1999; Bevins et al., 2002). Notably, the dopaminergic system is involved in mediating the conditioned rewarding effects of novelty (Besheer et al., 1999). Because alterations of the dopaminergic system and decreased reward functioning accompany nicotine withdrawal (and are likely not independent of each other), we expected that learning about reward would be altered during withdrawal. In the present experiment, we assessed the effects of nicotine withdrawal, at different time points, on novel-object place conditioning.

\section{Method}

Apparatus. Two similar three-compartment chambers were used in this experiment. The dimensions of the two end compartments were 31.0 $\mathrm{cm}$ long $\times 24.0 \mathrm{~cm}$ wide $\times 45.5 \mathrm{~cm}$ high; the smaller center compartment was $15.0 \mathrm{~cm}$ long $\times 24.0 \mathrm{~cm}$ wide $\times 45.5 \mathrm{~cm}$ high. One end compartment had black walls, a floor made of 13 metal rods, and a litter tray lined with newspaper. The other end compartment had white walls, a floor made of wire mesh, and a litter tray lined with pine wood chips. The center compartment had gray walls and an aluminum floor. The chamber floors were approximately $19.5 \mathrm{~cm}$ above the litter tray. During the habituation sessions and the postconditioning preference test, the inside walls of each end compartment were raised II $\mathrm{cm}$ to allow the rat free access to both end compartments.

Objects used in this task were a plastic scouring pad ( $9 \mathrm{~cm}$ diameter), a sponge ( $10 \mathrm{~cm}$ long $\times 7 \mathrm{~cm}$ wide $\times 3 \mathrm{~cm}$ high), a synthetic loofah (approximately $30 \mathrm{~cm}$ diameter), a sock (about $40 \mathrm{~cm}$ long), a white PVC pipe (16 $\mathrm{cm}$ long, $10.5 \mathrm{~cm}$ diameter opening), a plastic scouring pad ( $9 \mathrm{~cm}$ diameter) attached to a peach-colored paint roller ( $7.5 \mathrm{~cm}$ long, $4 \mathrm{~cm}$ diameter), and a sheet of newspaper wadded into a ball, presented in that order.

Procedure. Rats were assigned to one of the following groups $(n=$ 12 per group, except $n=16$ for the control group): control, WD1, WD2, WD3, or WD4. On Day 0, rats weighed on average $248 \pm 2$ g. On this day, rats in the WD groups were implanted with nicotine-filled pumps; rats in the control group were implanted with the empty pumps. Because of the weight gain across the 7 days, the actual nicotine dose delivered ranged from $10.32 \mathrm{mg} / \mathrm{kg}$ a day after the pump implantation to $8.74 \mathrm{mg} /$ $\mathrm{kg}$ on the 7 th day.

On Day 7, after a week of nicotine delivery, the pumps were removed for all the rats (i.e., spontaneous withdrawal for the nicotine-treated rats). For those rats in the WD groups, the number of days after pump removal is indicated by the number after the WD (e.g., WD1 = 1 day after pump removal). For example, rats in Group WD1 were tested in the novel-object place conditioning task 1 day after pump removal. Rats in the control group were divided into four subgroups so that 4 rats were tested in the task on each "withdrawal" day. This maneuver allowed us to assess performance at different durations from the pump removal surgery day.

Novel-object place conditioning task. Rats were allowed free access to both end compartments of the three-compartment chamber for $5 \mathrm{~min}$ to habituate to the apparatus. Rats were randomly assigned a paired compartment; the other compartment was designated unpaired. Rats were confined to each end compartment in an alternating pattern such that half of the rats in each group experienced the confinement order as paired-unpaired and the other half experienced the order as unpaired-paired. After each 5-min confinement, rats were returned to the home cage; there was a 30-min interval between each confinement. During each paired compartment confinement, a novel object was placed In the end compart-

Table 2

Median (IQR) Frequency of Somatic Signs Measured After Saline and Mecamylamine Injection

\begin{tabular}{lccccc}
\hline & \multicolumn{5}{c}{ Behavior } \\
\cline { 2 - 6 } Group & Abdom. & Eyeblinks & Facial fasc. & Wet-dog shakes & Misc. \\
\hline \multicolumn{7}{c}{ Saline } \\
Control & $3.5(2.5-5.5)$ & $2.0(1.0-2.0)$ & $0.0(0.0-1.0)$ & $3.0(2.0-4.0)$ & $3.0(1.5-4.5)$ \\
Nicotine & $5.0(2.0-6.5)$ & $2.0(0.5-2.5)$ & $0.0(0.0-1.0)$ & $3.5(1.5-4.5)$ & $2.5(1.5-4.5)$ \\
\hline \multicolumn{7}{c}{ Mecamylamine } \\
Control & $4.5(4.0-6.5)$ & $2.0(0.5-3.0)$ & $2.5(1.0-4.0)$ & $3.0(0.0-4.0)$ & $2.5(1.5-3.0)$ \\
Nicotine & $20.0^{*}(15.5-21.5)$ & $5.5 *(4.5-10.0)$ & $9.5 *(7.0-12.5)$ & $2.0(0.5-4.5)$ & $4.0(2.5-5.5)$ \\
\hline
\end{tabular}


ment. No object was present during unpaired compartment confinements. Rats received seven paired compartment confinements (i.e., $35 \mathrm{~min}$ total of novel object exposure) and seven unpaired compartment confinements. Which compartment (black or white) was paired with novelty was balanced in each group. A postconditioning test, identical to the initial habituation exposure, occurred approximately $30 \mathrm{~min}$ after the final confinement. During this test, rats had free access to both end compartments for $5 \mathrm{~min}$, and no objects were present in either compartment. This entire procedure was conducted in 1 day.

Behavioral observations and data analyses. The conditioning trials and the postconditioning test were videotaped for later observation of object interaction and compartment entries. Object interaction was defined as directed contacts with the object, excluding such contacts as backing into the object or bumping the tail against the object. To determine time spent in each end compartment during the postconditioning test, we recorded a compartment entry when the rat's forepaws were positioned in the compartment.

For each group, a difference score was calculated for the postconditioning test (i.e., total time spent in the paired compartment minus total time spent in the unpaired compartment). To assess whether the rats spent significantly more time in the paired compartment, we compared the difference score to a hypothetical median of $0 \mathrm{~s}$ by using the Wilcoxon signed rank test. A value significantly above $0 \mathrm{~s}$ indicates conditioning (i.e., more time spent in the novelty-paired compartment).

\section{Results and Discussion}

During the postconditioning test, 1 rat in the control group (tested 2 days after pump removal), 1 in the nicotine WD1 group, and 1 in the nicotine WD4 group did not sample both end compartments and were excluded from the analyses and figures. The behavior of the controls was compared across the four time delays (i.e., 1, 2, 3, and 4 days after pump removal). There were no significant differences for any of the behavioral measures, $\mathrm{Hs}<$ $6.433, p s>.92$. Accordingly, the rats were pooled into one group (control) for subsequent analyses and graphing.

Figure 2A illustrates the median difference score during the postconditioning test. There was a tendency for a significant group difference, $H(4)=9.46, p<.06$. To assess whether rats spent significantly more time in the novelty-paired compartment, the median of each group was compared with a hypothetical value of $0 \mathrm{~s}$. Recall that a difference score significantly above $0 \mathrm{~s}$ indicates conditioning. Conditioning was only observed in the control group and Group WD4, with both groups spending significantly more time in the novelty-paired compartment during the test $(p s<.01)$. Nicotine withdrawal blocked conditioning 1,2 , and 3 days after cessation of nicotine administration. That is, rats in Groups WD1, WD2, and WD3 did not display difference scores significantly greater than $0 \mathrm{~s}(p \mathrm{~s}<.17)$.

Recall that in Experiment $1 \mathrm{~A}$, an overt withdrawal reaction was observed up to 3 days after removal of the osmotic pump. The occurrence of these behaviors might compete with general locomotor activity and affect performance in the nicotine withdrawal groups (e.g., WD1, WD2, and WD3). Several studies have shown no alteration of locomotor activity using withdrawal parameters similar to those used in the present study (Helton, Modlin, Tizzano, \& Rasmussen, 1993; Hildebrand et al., 1999; Irvine, Cheeta, \& File, 1999; Malin et al., 1992; but see Fung et al., 1996). Further, we directly assessed this possibility in our situation by using the number of compartment entries during the postconditioning test as a measure of general locomotor activity. Fig-
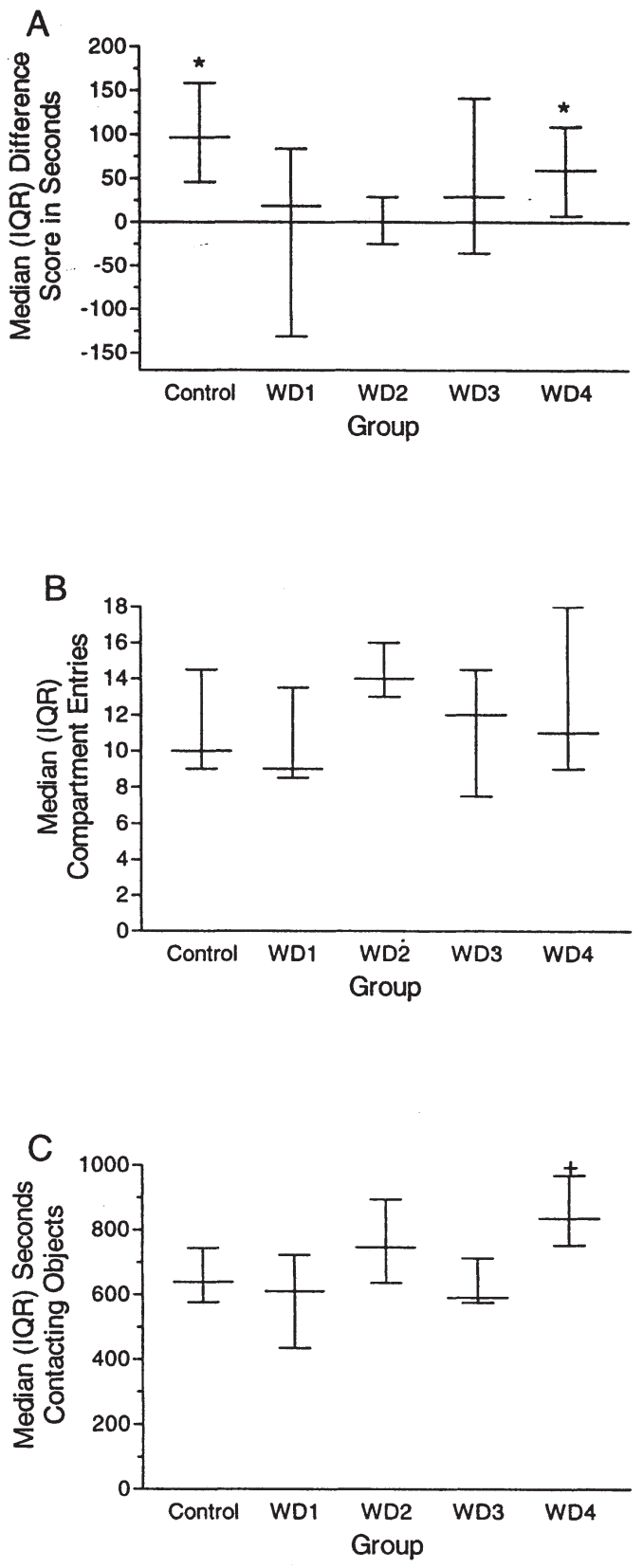

Figure 2. A: Median and interquartile range (IQR) of the difference score (time in paired compartment minus time in unpaired compartment) during the postconditioning preference test, over the 4 days after pump removal (withdrawal days [WD]). A value above the line drawn at $0 \mathrm{~s}$ indicates conditioning (i.e., more time spent in novelty-paired compartment). Asterisks indicate a significant difference from a hypothetical median of $0 \mathrm{~s}(p<.05)$. B: Median and IQR of compartment entries during the postconditioning preference test. C: Median and IQR of object interaction during the conditioning phase. Plus sign indicates a significant difference from the control group $(p<.05)$.

ure $2 \mathrm{~B}$ shows the median compartment entries during the postconditioning preference test. Nicotine withdrawal did not affect locomotor activity as assessed by compartment entries during the postconditioning test, $H(4)=8.88, p=.064$.

Another possibility is that withdrawal suppressed object interaction in Groups WD1, WD2, and WD3. For example, if rats 
undergoing withdrawal did not interact with the objects, a conditioned association would presumably not develop because novelty was not experienced. Figure $2 \mathrm{C}$ shows the median total time (in seconds) spent interacting with the objects during the conditioning phase. A significant group difference was revealed, $H(4)$ $=17.34, p=.002$, with Group WD4 spending more time interacting with the objects than controls $(p<.05)$. This result is intriguing, given that withdrawal did not affect object interaction in any of the other groups. Replication of this data pattern will merit further investigation into possible mechanisms mediating stimulated interaction after a period of withdrawal. However, the conclusion of greatest interest is that the blockade of conditioning in Groups WD1, WD2, and WD3 cannot be attributed to altered motoric behavior as measured by object interaction and compartment entries.

The major finding from this experiment was the blockade of conditioning during the early stages of nicotine withdrawal (i.e., 1, 2 , or 3 days after pump removal). Given the previous research indicating decreased reward functioning during withdrawal and our previous research implicating the dopamine system in mediating the acquisition of the conditioned association, one explanation for this data pattern is that nicotine withdrawal blocked the formation of the conditioned association. This withdrawal-induced blockade may have been due to decreased rewarding properties of interacting with the objects, and/or the alterations in dopamine transmission may have prevented the formation of a conditioned association. Before accepting such an explanation, however, there are several alternative possibilities that require empirical attention:

1. Nicotine withdrawal might affect processing of the information about the novel objects. An impairment of this nature would compromise learning about each of the objects and this would result in the failure to develop a conditioned association.

2. Neural processes required for environmental familiarization might be altered during the withdrawal state. For example, if the stimulus information about each environment (e.g., floor texture, odor, brightness) was not processed, the two compartments would appear novel during each confinement and testing. Further, this interference would prevent the formation of an association between the novel objects and the specific environment in which they are paired.

3. Withdrawal might affect expression of the conditioned association. That is, the acquisition of the conditioned association was intact (which would imply that the rewarding properties of the novel stimulation were not altered), but the processes required to express the conditioned association were impaired. The following experiments examined these alternative explanations.

\section{Experiment 3}

As noted previously, spontaneous nicotine withdrawal may have affected the neural processing of information about the novel objects. To test this possibility directly, we used a preparation that takes advantage of a rat's tendency to interact more with a novel object than a previously experienced (familiar) object (Berlyne, 1950). Detection of a novel object in the environment presumably requires intact neural processing mechanisms for rats to discriminate between the two objects during the test. For example; in our laboratory, we have consistently found that rats that receive exposure to a sample object for $10 \mathrm{~min}$ spend more time interacting with the novel object than the sample object $1 \mathrm{hr}$ later (Besheer et al., 1999; Besheer \& Bevins, 2000b; Besheer, Short, $\&$ Bevins, 2001). This discrimination suggests that the rat processed the information about the stimulus properties of the sample object (e.g., texture, size, odor) during sample-object exposure and displayed the learning by spending more time with the novel object during the novel-object test. Sufficient exposure to the sample object is necessary in order for the rat to detect the novel object during the test. For example, after only 2 min of sample-object exposure, rats do not discriminate between the sample and the novel object during the novel-object test (Besheer \& Bevins, 2000b).

If undergoing withdrawal affected neural processing of the novel objects in the previous experiment, we would expect to observe a failure in novel-object detection in the present experiment. That is, during sample-object exposure, rats would interact with the object, but a failure in processing stimulus information about the object would produce a subsequent failure to discriminate between the objects because both objects would appear as novel to the rat during the test.

\section{Method}

Apparatus and procedure. The three-compartment chamber detailed in Experiment 2 was used in this experiment. Rats were assigned to one of the following groups: control, WD1, WD2, WD3, or WD4 $(n=8$ per group, except $n=16$ for the control group). On Day 0, rats in the WD groups were implanted with the nicotine-filled pumps. Rats in the control group were implanted with the empty pumps. The average weight of the rats on the surgery day was $259 \pm 2 \mathrm{~g}$. On Day 7 , after 1 week of nicotine delivery, the pumps were removed for all the rats. The approximate nicotine dose delivered during this time ranged from $10.42 \mathrm{mg} / \mathrm{kg}$ a day after the pumps were implanted to $9.32 \mathrm{mg} / \mathrm{kg}$ on the day of pump removal. As in Experiment 2, for those rats in the nicotine withdrawal groups (i.e., WD groups), the number of days between pump removal and behavioral testing is denoted by the number following the WD. Rats in the control group were assigned to four subgroups such that 4 rats participated in the novel-object detection task on each withdrawal day to control for the task occurring at different durations after the pump removal surgery day in the experimental conditions.

Novel-object detection task. Rats were confined to one end compartment of the three-compartment chamber with a sample object for 3 min and immediately transferred with the same object to the other end compartment for another $3 \mathrm{~min}$. Rats were then returned to the home cage. The novel-object test occurred I hr later. During this test, rats were allowed free access to both end compartments for $2 \mathrm{~min}$. A novel object was present in one end compartment, and the previously experienced sample object was present in the other end compartment. The novel object, the compartment in which initial placement with the sample object occurred, and the compartment in which the novel object was placed were balanced as much as allowed by the sample size. The object pair was the $10-\mathrm{cm}$ sponge and a white PVC pipe (15 cm long, $5 \mathrm{~cm}$ opening).

Behavioral observations and data analyses. The novel-object test was videotaped for later observation of object interaction and compartment entries. A difference score for each group was calculated for the novel-object test (i.e., time spent interacting with novel object minus time spent interacting with sample object). To assess whether rats discriminated between the two objects, we used the Wilcoxon signed rank test to compare the difference score with a hypothetical median of $0 \mathrm{~s}$. A value significantly larger than $0 \mathrm{~s}$ indicates more time spent with the novel object (see Besheer \& Bevins, 2000b; Besheer et al., 2001). 


\section{Results and Discussion}

Recall that out of the 16 rats implanted with the empty pumps, 4 were tested on each "withdrawal" day. These rats did not statistically differ on any dependent measure that was assessed ( $H \mathrm{~s}<6.57, p \mathrm{~s}>.09)$; thus the rats were pooled into one group (control) for subsequent analyses and graphing.

Figure 3A illustrates the median difference score for each group. Nicotine withdrawal did not affect time spent with the novel object, as indicated by similar difference scores across groups, $H(4)=0.85, p=.93$. Novel-object discrimination was

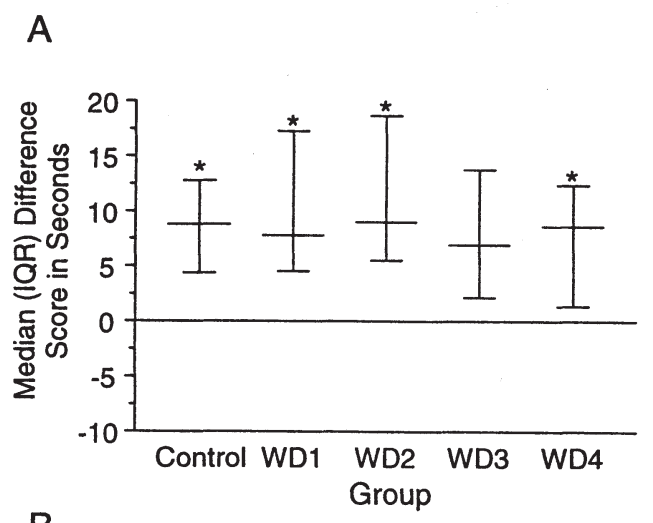

B

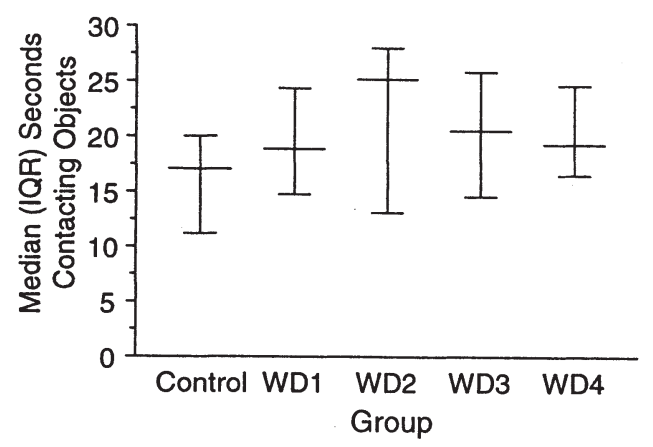

C

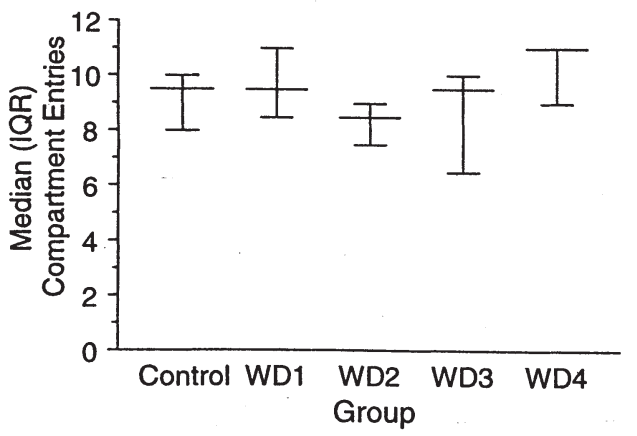

Figure 3. A: Median and interquartile range (IQR) of the difference score (time with novel object minus time with sample object) for each group during the novel-object test, over the 4 days after pump removal (withdrawal days [WD]). A value above the line drawn at $0 \mathrm{~s}$ indicates a preference for the novel object. Asterisks indicate a significant difference from a hypothetical median of $0 \mathrm{~s}(p<.05)$. B: Median and IQR of time spent interacting with both the novel and sample object during the novel-object test. C: Median and IQR of the number of compartment entries during the novel-object test. observed in the control, WD1, WD2, and WD4 groups, as indicated by median difference scores significantly greater than $0 \mathrm{~s}(p \mathrm{~s}<.04)$. In Group WD3, there was a weak tendency for a blockade of novel-object detection $(p<.06)$. That is, time spent with the novel object did not differ significantly from $0 \mathrm{~s}$. This data pattern may be a result of a statistical Type II error. Regardless, withdrawal did not reliably affect detection of the novel object. The median number of seconds spent interacting with the novel and sample objects' and the median number of compartment entries during the novel-object test are illustrated in Figures $3 \mathrm{~B}$ and $3 \mathrm{C}$, respectively. Nicotine withdrawal did not affect object interaction, $H(4)=4.69, p=$ .32 , or the number of compartment entries, $H(4)=4.51, p=$ .34 , indicating no impairment in motor functioning.

In sum, spontaneous nicotine withdrawal did not reliably affect novel-object detection. We take this result to indicate that neither processing of the information about the sample object (e.g., texture, odor) nor the neural consolidation of that information were impaired. This result further implies that the blockade of conditioning in the prior experiment (at least in Groups WD1 and WD2) was not due to a failure to process the information about the novel objects.

\section{Experiment 4}

Another plausible explanation for the failure to observe conditioning in the novel-object place conditioning task is that withdrawal affected environmental familiarization processes. That is, if the rat's ability to familiarize with the environment was impaired, then the formation of an association between the novel objects and the environment in which those objects were repeatedly presented would also be impaired. As a result, rats would not discriminate between the two compartments during the postconditioning test. The preparation used to assess this familiarization account takes advantage of a rat's tendency to interact more with a novel object in a familiar environment than in an unfamiliar/novel environment (Bevins, Koznarova, \& Armiger, 2001). Briefly, during the environmental familiarization phase, rats were exposed to an environment for a specified amount of time. Another group was not exposed to the environment during this phase. On the following day, all rats were placed into the environment along with a novel object. Rats previously exposed to the environment displayed greater object interaction than rats that had not been previously exposed to the environment. Bevins, Koznarova, and Armiger (2001) concluded that object interaction can serve as an index of environmental familiarization (cf. Sheldon, 1969).

To assess environmental familiarization during nicotine withdrawal, we used this preparation with an enclosed, rather than an open and elevated, environment. This apparatus modification was made to reduce any anxiogenic properties of the elevated environment and to reduce competing behaviors, such as "extra-platform" exploration, that may interfere with familiarization (see Bevins, Koznarova, \& Armiger, 2001). Also, nicotine withdrawal was precipitated by an injection of mecamylamine before the environmental familiarization phase so that only this phase occurred during withdrawal. 


\section{Method}

Apparatus. The apparatus used was a modified version of the threecompartment chamber used in Experiments 2 and 3. The dimensions of the chamber and the flooring in the gray center compartment were the same as described previously, except that the chamber floors were approximately $3 \mathrm{~cm}$ above the litter tray. The flooring in both end compartments was metal rods, the chamber walls were painted white, and both litter trays were lined with newspaper. The scouring pad attached to the paint roller (see Experiment 2) was the object used during the test.

Procedure. On Day 0, rats underwent osmotic pump implantation surgery. Because of weight gain across the 7 days, the actual nicotine dose delivered ranged from $10.08 \mathrm{mg} / \mathrm{kg}$ a day after the pump implantation to $9.15 \mathrm{mg} / \mathrm{kg}$ on the 7 th day. Rats in the control group were implanted with the sealed pipet bulb, as described in Experiment 1B. On the day of pump implantation, rats weighed an average of $256 \pm 2 \mathrm{~g}$.

For the environmental familiarization phase, rats from each condition (i.e., nicotine and saline) were assigned to Group Familiar (nicotine $n=8$, control $n=8$ ) or Group Unfamiliar (nicotine $n=8$, control $n=$ 8). On Day 6, all rats in Group Familiar were exposed to the apparatus for 2 min. Placement of the rats (left or right compartment) was balanced. Rats in Group Unfamiliar were treated like those in Group Familiar: They were transferred to the experimental room in the home cage, were removed from the cage as if they were going to be placed in the apparatus, but were instead returned to the home cage. The entire procedure was repeated 30 min later. Thus, rats in Group Familiar received 4 min of environmental familiarization; Group Unfamiliar received equivalent handling and transport experience.

All rats in the nicotine condition, Group Familiar and Group Unfamiliar, were injected subcutaneously with mecamylamine before the first environmental familiarization placement (i.e., mecamylamine-precipitated withdrawal) and with saline before the second placement. In the control condition, half of the rats in Group Familiar and half of the rats in Group Unfamiliar were injected with mecamylamine before the first placement and saline prior to the second placement. The remaining rats were injected with saline prior to both placements. The injections always occurred $10 \mathrm{~min}$ before the placement.

The test phase occurred 1 day after the initial environmental familiarization session. All rats received a subcutaneous saline injection 10 min prior to placement in the chamber for $2 \mathrm{~min}$. Recall that for Group Unfamiliar, this was the first exposure to the environment. Initial compartment placement was balanced so that half of the rats from each condition started on each side. For Group Familiar, each rat was placed on the same side as during the environmental familiarization phase. The object was placed on the end opposite from rat placement. This object was novel to all the rats.

Behavioral observations and data analyses. All sessions were videotaped for later behavioral observations. The number of line crosses into each side of the chamber was measured during environmental familiarization and the novel-object test. A line cross was recorded when the rat's forepaws crossed a line that was drawn on the monitor dividing the chamber in half (i.e., bisected the center compartment). The latency to make the initial line cross was also measured. A latency of $120 \mathrm{~s}$ was recorded if the rat did not make a line cross. During the novel-object test, time spent interacting with the object and latency to make the first contact was measured. Object interaction was scored as previously described. Planned a priori contrasts (Dunn's multiple comparison tests) prompted by a significant Kruskal-Wallis test were made to assess whether each condition (control or nicotine) in Group Familiar differed from the comparable condition in Group Unfamiliar and whether nicotine withdrawal affected behavior as assessed by the various dependent measures (i.e., control vs. nicotine condition in Group Familiar).

\section{Results and Discussion}

Recall that in the control condition, half of the rats in Groups Familiar and Unfamiliar received a mecamylamine injection prior to environmental familiarization; the remaining rats received saline. Treating a subset of rats with mecamylamine allowed us to assess whether the drug had any nonspecific effects on behavior. During the environmental familiarization phase and the test phase, the saline- and mecamylamine-treated controls in Group Familiar did not differ statistically on any of the dependent measures collected $\left(U_{\mathrm{s}}<6.5\right.$, ps $>$.48). The saline; and mecamylamine-treated controls in Group Unfamiliar did not differ on any of the measures during the test phase $\left(U_{\mathrm{s}}=7, p \mathrm{~s}<.89\right)$. Because mecamylamine did not significantly affect behavior, the data from the rats were pooled. Thus, the controls of Group Familiar and Group Unfamiliar each included 8 rats, 4 that had received a saline injection and 4 that had received a mecamylamine injection.

During the familiarization phase, the number of line crosses and latency to make the initial line cross from both familiarization placements were averaged for each rat (cf. Bevins, Koznarova, \& Armiger, 2001). Recall that only Group Familiar (both nicotine and control conditions) experienced environmental familiarization. Nicotine withdrawal did not affect the locomotor activity as measured by the number of line crosses $(U=28, p<.73)$ or the latency to make the initial line cross during each placement $(U=26, p<.58$; data not shown). Thus, nicotine withdrawal did not affect activity as expressed by these measures.

Figure 4A shows object interaction during the test phase for each group. Recall that for Groups Unfamiliar, the test phase was the first exposure to the environment. A significant group difference in object interaction was revealed, $H(3)=$ $11.92, p=.008$. Consistent with previous research, in the control (no withdrawal) condition, rats in Group Familiar interacted more with the object than rats in Group Unfamiliar $(p<$ $.05)$. The same data pattern was observed in the nicotine conditions $(p<.05)$. Nicotine withdrawal did not affect environmental familiarization processes, as rats in the nicotine condition of Group Familiar did not differ in object interaction from the controls of Group Familiar $(p>.05)$. Similarly, as illustrated in Figure 4B, a significant difference in the latency to make the first contact with the object was observed, $H(3)=$ $14.49, p=.002$. Rats in Group Familiar were faster to make the initial object contact than rats in Group Unfamiliar, regardless of condition (control or nicotine; $p \mathrm{~s}<.05$ ). Also, precipitating withdrawal prior to environmental familiarization did not affect the expression of this behavior, as the latency to make initial object contact did not differ between the nicotine and control conditions in Group Familiar $(p>.05)$.

Figure 4C illustrates the total number of line crosses during the test. There was a significant group difference, $H(3)=$ $13.79, p=.003$, with control rats in Group Familiar making more line crosses than control rats in Group Unfamiliar $(p<$ $.05)$. In contrast to the other measures, in the nicotine condition, there was no significant difference between the number 

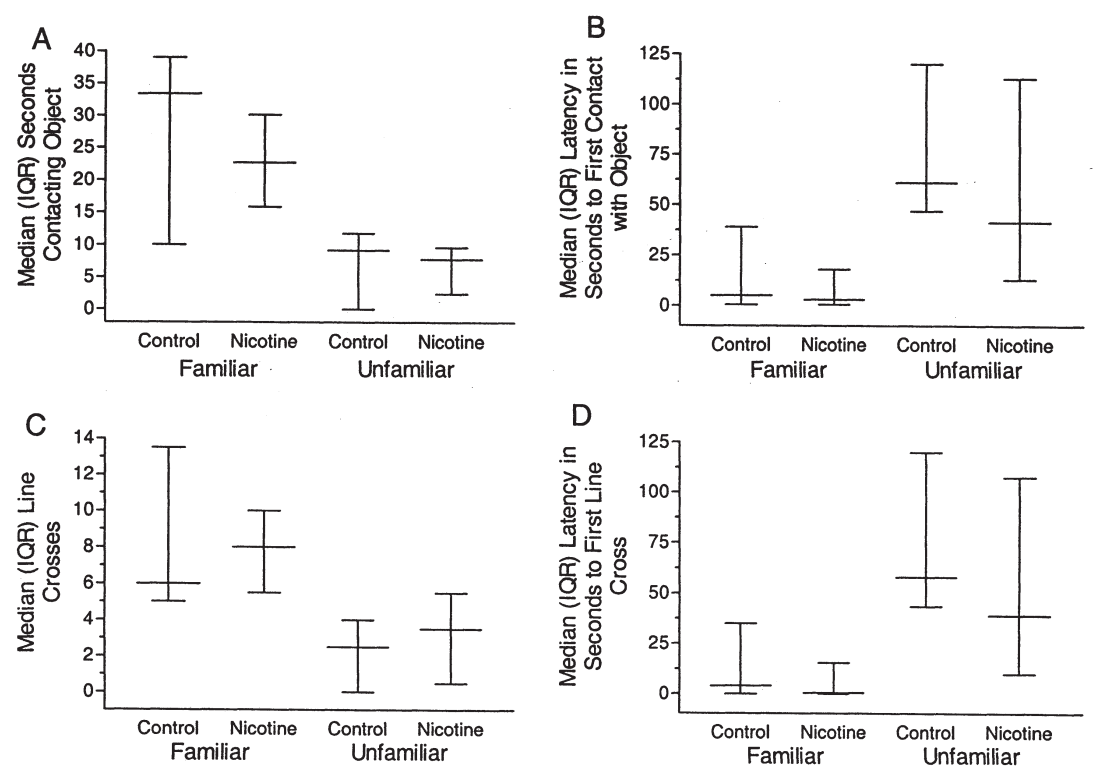

Figure 4. A: Median and interquartile range (IQR) of object interaction during the test phase. B: Median and IQR of the latency to make the initial contact with the object during the test phase. C: Median and IQR of the number of line crosses during the test phase. D: Median and IQR of the latency to make the initial line cross during the test phase.

of line crosses between Groups Familiar and Unfamiliar ( $p$ $>.05)$. As illustrated in Figure 4D, a significant difference in the latency to make the initial line cross was observed, $H(3)$ $=14.63, p=.002$. Rats in both conditions in Group Familiar were faster to make the initial line cross than the comparable condition in Group Unfamiliar $(p s<.05)$. Finally, the nicotine and control conditions in Group Familiar did not differ, indicating that nicotine withdrawal again did not affect the expression of this behavior $(p>.05)$.

These results replicate previous work showing that object interaction can serve as a measure of environmental familiarization (Bevins, Koznarova, \& Armiger, 2001; Sheldon, 1969). Nicotine withdrawal did not affect environmental familiarization processes. Rats that experienced nicotine withdrawal during the familiarization phase interacted with the novel object as much as the controls that had also previously experienced the environment. If nicotine withdrawal had affected familiarization processes, we would have expected the deficit to be expressed as a decrease in object interaction (i.e., as if the environment had not been previously experienced; see Groups Unfamiliar). In addition to unaltered object interaction, the other measures of environmental familiarization such as latencies and line crosses were also generally not affected by withdrawal. Together these results do not support the explanation that the failure to observe a preference for the novelty-paired compartment in the novel-object place conditioning preparation (Experiment 2) was due to impairment of familiarization processes by nicotine withdrawal.

\section{Experiment 5}

In Experiment 2, rats undergoing spontaneous nicotine withdrawal did not display a conditioned preference for the novelty-paired environment. That impairment was not a result of a deficit in processing the information about the objects because novel-object detection was not affected by spontaneous withdrawal (Experiment 3). An account based on a failure to process environmental stimuli was also eliminated given that nicotine withdrawal did not alter environmental familiarization processes (Experiment 4). The present experiment was conducted to evaluate whether experiencing nicotine withdrawal during the postconditioning test would block the expression of preference for the novelty-paired environment. That is, conditioning proceeded unaltered so as not to interfere with the formation of the conditioned association. Withdrawal was precipitated before the postconditioning test. A blockade in novel-object place conditioning during the postconditioning test would suggest that nicotine withdrawal interfered with expression, rather than acquisition, of a conditioned association.

\section{Method}

Apparatus. The three-compartment chamber and objects described in Experiment 2 were used in the present experiment. Rats were assigned to one of the following groups: control-saline (Control-Sal, $n=10)$, control-mecamylamine (Control-Mec, $n=10$ ), nicotine-saline (Nic-Sal; $n=$ 12), or nicotine-mecamylamine (Nic-Mec, $n=15$ ).

Procedure. On Day 0, the day of pump implantation, rats weighed an average of $289 \pm 6 \mathrm{~g}$. Each rat in the control group was implanted with the sealed bulb, and each rat in the nicotine groups was implanted with a nicotine-filled osmotic pump. Because of weight gain across the 7 days, the nicotine delivered ranged from $10.22 \mathrm{mg} / \mathrm{kg}$ a day after the pump implantation to $9.40 \mathrm{mg} / \mathrm{kg}$ on the 7 th day of nicotine delivery.

The procedures for the novel-object place conditioning task were the same as described in Experiment 2. Briefly, on Day 7, rats received repeated confinement to both end compartments of the chamber; one was 
paired with a novel object, the other was not. Twenty minutes before the final 2 confinements (i.e., the 13th and 14th confinements), rats in all groups were injected subcutaneously with saline. Similarly, 20 min prior to the postconditioning test, rats in Groups Control-Sal and Nic-Sal received a saline injection; rats in Groups Control-Mec and Nic-Mec received a $1 \mathrm{mg} / \mathrm{kg}$ mecamylamine injection. Thus, withdrawal was precipitated prior to the test in Group Nic-Mec.

\section{Results and Discussion}

Group Control-Mec was included in the experiment to assess any nonspecific effects of mecamylamine. Groups Control-Sal and Control-Mec did not differ on any of the measures $(U \mathrm{~s}>29.5, p \mathrm{~s}>.12)$, indicating that mecamylamine did not have any nonspecific effects on the behaviors of interest. Accordingly, the rats were pooled into one group termed Group Control $(n=20)$.

During the postconditioning test, the difference scores did not differ across the groups, $H(2)=2.94, p=.23$ (see Figure $5 \mathrm{~A})$. Consistent with the results of Experiment 2, rats that received novel objects paired with an end compartment spent more time in that compartment during the postconditioning test (Group Control), $p=.0009$. Similarly, rats in both nicotine groups (Nic-Sal, Nic-Mec) spent significantly more time in the novelty-paired compartment $(p \mathrm{~s}<.03)$. That is, nicotine withdrawal precipitated before the test did not significantly alter the expression of the conditioned preference. Figure $5 \mathrm{~B}$ shows the median object interaction during the conditioning confinements. There was a significant group difference in object interaction, $H(2)=9.33, p<.01$, with both nicotine groups interacting with the objects more than the control group. This result is consistent with previous work showing chronic nicotine-induced locomotor stimulation in other preparations (Bevins, Besheer, \& Pickett, 2001; Clarke \& Kumar, 1983; Ksir, Hakan, \& Kellar, 1987). In contrast, compartment entries during the postconditioning test did not differ across groups, $H(2)=1.66, p<.44$ (see Figure 5C). This apparent discrepancy in locomotor stimulation may be explained by the fact that the test occurred in a free-choice situation and was only $5 \mathrm{~min}$ in length. That is, the 5 -min test was a relatively brief period of time during which the rats shuttled between compartments compared with the seven 5-min repeated confinements with novel objects. In addition, recall that for Group Nic-Mec, this postconditioning test occurred during nicotine withdrawal, thus only Group Nic-Sal was experiencing nicotine during the test.

The main findings of this experiment are that chronic nicotine delivery during conditioning and the postconditioning test did not affect learning about the novelty-paired compartment (i.e., Group Nic-Sal), and that nicotine withdrawal precipitated prior to the test did not alter the expression of the conditioned association for the novelty-paired compartment (i.e., Group Nic-Mec). The finding that chronic nicotine did not enhance time spent in the novelty-paired compartment is interesting given that nicotine has been found to facilitate learning in several behavioral preparations (Abdulla, Calaminici, Stephenson, \& Sinden 1993; Besheer \& Bevins, 2000a; Levin et al., 1990, Mirza \& Stolerman, 1998). However, to our know-
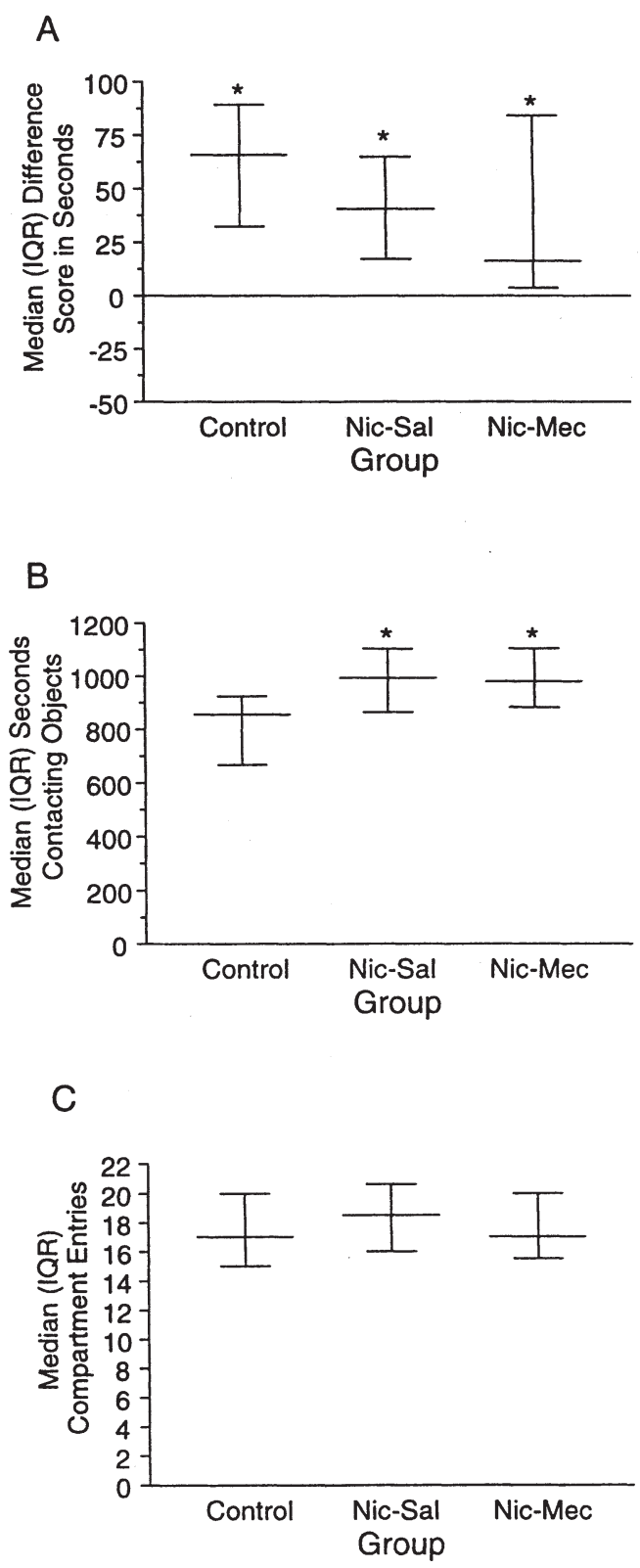

Figure 5. A: Median and interquartile range (IQR) of the difference score (time in paired compartment minus time in unpaired compartment) during the postconditioning test. A value above the line drawn at $0 \mathrm{~s}$ indicates a preference for the novelty-paired compartment. Asterisks indicate a significant difference from a hypothetical median of $0 \mathrm{~s}(p<.05)$. B: Median and IQR of object interaction during the conditioning phase. Asterisks indicate a significant difference from the control group $(p<.05)$. $\mathrm{C}$ : Median and IQR of compartment entries during the postconditioning preference test. $\mathrm{Nic}=$ nicotine; $\mathrm{Sal}=$ saline; $\mathrm{Mec}=$ mecamylamine.

ledge, a nicotine-enhancing effect in a place conditioning task not involving nicotine as the appetitive outcome has not been reported. A possible reason that enhanced performance was not observed in this experiment (Group Nic-Sal) is that place conditioning procedures tend not to show graded functions. For example, the data patterns commonly reported in place conditioning experiments are the presence or absence of a place preference or aversion (see Bardo \& Bevins, 2000). 
The result of most interest is that nicotine withdrawal precipitated before the test did not influence time spent in the novelty-paired environment. Specifically, the expression of the conditioned association was unaffected, as rats spent more time in the compartment that had been paired with novel objects. Presumably, the preference for the novelty-paired compartment observed in this experiment is a result of the formation of a conditioned association between the rewarding properties of interacting with the novel objects and the environment in which novelty was paired. These results help interpret the findings of Experiment 2. In that experiment, rats experienced withdrawal during conditioning (acquisition phase), as well as during the test (expression phase). The results from the present experiment suggest that withdrawal during the test was not a major contributing factor for the failure to display the paired-compartment preference. A more likely explanation is that nicotine withdrawal impaired the acquisition of the association between the reward and the environment in which it was experienced.

\section{General Discussion}

The major goal of this study was to evaluate the notion of altered reward functioning during nicotine withdrawal by using a novel-object place conditioning preparation. Previous work has found that access to novel objects is rewarding (Besheer et al., 1999; Bevins \& Bardo, 1999; Bevins et al., 2002). That is, in a 4day (Besheer et al., 1999; Bevins \& Bardo, 1999) and an 8-day (Bevins et al., 2002) conditioning procedure, rats display an increase in preference for an environment repeatedly paired with novel objects. The present report extends the generality of novel-object place conditioning to include a 1-day procedure. Further, this single-day protocol can be a useful tool in a variety of research areas to measure short-lasting or time-dependent effects. For example, conditioning can be assessed during critical developmental periods, or during different stages of withdrawal from other drugs of abuse.

Notably, spontaneous nicotine withdrawal blocked conditioning to an environment reliably paired with access to novel objects 1,2 , and 3 days after pump removal. This blockade was time dependent, as by the 4th day after pump removal, the preference reemerged. We concluded that this blockade was due to the decreased rewarding properties of interacting with the objects and/or that the alterations in dopamine transmission prevented the formation of a conditioned association. This conclusion was strengthened by the fact that we empirically assessed alternative accounts and found that nicotine withdrawal did not affect processing of the information about the objects, environmental familiarization, or expression of the conditioned association. The elimination of each of these alternative explanations allowed us to conclude that these factors were unlikely to have contributed to the blockade in place conditioning.

There exist at least two tenable explanations for the failure to acquire the conditioned association. In the novel-object place conditioning preparation, an association is presumably formed between the environment and the rewarding properties of interacting with the novel objects. The first account predicts that the neurochemical changes that occur during the early stages of nicotine withdrawal, specifically the changes in dopamine functioning, interfered with the formation of the conditioned association.
As discussed earlier, we have previously found that antagonism of the dopamine $\mathrm{D}_{1}$ receptor subtype by $\mathrm{SCH}-23390$ blocks the acquisition of novel-object place conditioning (Besheer et al., 1999). That research parallels other work involving the $D_{1}$ receptor in the acquisition of drug-conditioned place preferences (Cervo \& Samanin, 1995; Shippenberg \& Herz, 1988; Sutton \& Beninger, 1999). One theory of reward-related learning suggests that an intact signal at the $\mathrm{D}_{1}$ receptor is necessary for the rewarding properties of the stimulus to be associated with the environment in which the stimulus is repeatedly paired (Sutton \& Beninger, 1999). From this perspective, altered dopamine functioning during the early phases of nicotine withdrawal interferes with formation of the conditioned association by disrupting the signal at the $\mathrm{D}_{1}$ receptor subtype.

In previous work, we reported a dissociation between the processes that mediate novel-object place conditioning and those that mediate novel-object detection (Besheer et al., 1999; Bevins, 2001). Blockade of $D_{1}$ prior to each novel-object pairing blocked place conditioning, whereas $\mathrm{D}_{1}$ antagonism prior to the novel-object test in the novel-object detection preparation did not affect novelty detection. Further testing of the $\mathrm{D}_{2}, \mathrm{D}_{3}$, and $\mathrm{D}_{4}$ receptor subtypes also failed to find a role for dopamine in novelty detection (Besheer et al., 2001). We concluded that the processes mediating a conditioned association with novelty differ from those that mediate the detection of novelty. The results of the present study lend further support to this conclusion. That is, nicotine withdrawal induced prior to the novel-object test in the detection task did not affect detection of the novel object; withdrawal induced prior to conditioning, however, blocked acquisition of a novel-object conditioned place preference.

The second explanation of the withdrawal-induced blockade of conditioning is based on previous work showing altered reward functioning during nicotine withdrawal (e.g., elevated ICSS thresholds; Bozarth et al., 1998; Epping-Jordan et al., 1998; Harrison, Liem, \& Markou, 2001). This decrease in reward functioning is taken to indicate a state of anhedonia, in which the impact of rewarding stimuli is reduced (Epping-Jordan et al., 1998; Harrison et al., 2001). This withdrawal-induced anhedonia account predicts that the rewarding effects of interacting with the novel objects are reduced, thus impairing the formation of the conditioned association (i.e., decreased reward magnitude). This account suggests that the quality of object interaction differs between "normal" control rats and rats experiencing nicotine withdrawal. That is, object interaction acquires or includes a rewarding quality (as evidenced by conditioning) in control rats, but not in rats undergoing withdrawal. However, the quantity of object interaction is maintained in rats experiencing withdrawal (see below). The novel-object place conditioning preparation was introduced to the literature by Bevins and Bardo (1999). In that report, the authors hypothesized that part of the time spent interacting with the object during each conditioning session likely reflects some of the object's rewarding value. According to this assumption, the withdrawal-induced anhedonia account would predict object interaction to be decreased in rats undergoing withdrawal. That is, because object interaction does not acquire or contain a rewarding quality, rats experiencing withdrawal would not interact with the objects as much as the controls. This predicted data pattern was not observed in the present work, suggesting that object interaction might not serve as an index of the re- 
warding value of novelty (see Besheer et al., 1999). Regardless, it is intriguing that rats experiencing withdrawal, presumably in a state of anhedonia, interacted with the objects as much as controls. If the rewarding properties of interacting with novelty are decreased in rats experiencing withdrawal, then what maintains object interaction?

In the experiments presented in this report, object interaction is an exploratory response (cf. Berlyne, 1950). Presumably, during each confinement with the novel object, the rat approaches the object in order to explore the various stimulus properties of the object, such as size, texture, and odor. Recall that the rat was allowed 5 min (length of conditioning trial) to explore each distinct novel object. In our laboratory, we have repeatedly observed that, in a 10-min session with an object, interaction is higher during the first $5 \mathrm{~min}$. Presumably, the object loses some of its novelty across time, as indicated by decreased object interaction in the second half of the 10-min session (see Berlyne, 1950). Thus, in the relatively short exposures used in the present place conditioning protocol ( $5 \mathrm{~min}$ ), the novelty of each different object was likely maintained. Further, in this study we have determined that experiencing withdrawal does not affect the detection of novelty. consequently, rats undergoing withdrawal approach and interact with the object to explore the novelty in the environment. In the control rats, the object exploration and interaction presumably acquire or include a rewarding quality; however, in rats experiencing anhedonia, this exploratory response may not acquire or include a rewarding quality as a result of reward functioning blunted by withdrawal.

Notably, rats that were tested in this preparation on the 4th day after cessation of chronic nicotine delivery displayed a novelty-conditioned association and an increase in object interaction above control levels. This data pattern can be readily explained by a withdrawal-induced anhedonia account. After a period of decreased reward functioning as experienced during the early stages of withdrawal, reward functioning gradually returns to normal. This pattern is evident in the ICSS literature, as indicated by reward thresholds that return to baseline levels between $48 \mathrm{hr}$ (Harrison et al., 2001) and $128 \mathrm{hr}$ (Epping-Jordan et al., 1998) after the cessation of chronic nicotine delivery. One account, albeit speculative, is that restored reward functioning after a period of anhedonia may enhance the rewarding properties of interacting with novelty (i.e., contrast effect). However, given that the place conditioning preparation is not highly sensitive to increases in reward value (see Bardo \& Bevins, 2000), a greater conditioning score would not be expected in this group.

The altered dopamine transmission account and the withdrawal-induced anhedonia account are tenable explanations for the failure to observe a conditioned association in the novel-object place conditioning procedure. The results from the present study do not allow dissociation between the accounts. However, it is important to note that decreased reward functioning and altered dopamine transmission are not necessarily mutually exclusive. In fact, the decreases in dopamine transmission that accompany withdrawal from nicotine and various other drugs of abuse are likely directly related to changes in reward functioning (cf. Carboni et al., 2000; Hildebrand et al., 1999; Stinus, Le Moal, \& Koob, 1990). Given the clear negative health consequences of tobacco use, there is an urgent need to find more effective methods for maintaining abstinence (i.e., new pharmacotherapies, be- havioral treatments). Consequently, further investigation into the neurochemical and behavioral changes that accompany nicotine withdrawal will lead to a better understanding of the withdrawal syndrome. The present study contributes to the literature anew method by which to evaluate the affective components of drug withdrawal. In addition to the ICSS preparation, this novel-object place conditioning task could be a useful tool to investigate the efficacy of various pharmacotherapies.

\section{References}

Abdulla, F. A., Calaminici, M.-R., Stephenson, J. D., \& Sinden, J. D. (1993). Chronic treatments with cholinoceptor drugs influence spatial learning in rats. Psychopharmacology (Berlin), 111, 508-511.

Adams, M. L., \& Cicero, T. J. (1998). Nitric oxide mediates mecamylamine- and naloxone-precipitated nicotine withdrawal. European Journal of Pharmacology, 345, RI-R2.

Bardo, M. T. , \& Bevins, R. A. (2000). Conditioned place preference: What does it add to our preclinical understanding of drug reward? Psychopharmacology (Berlin), 153, 31-43.

Berlyne, D. E. (1950). Novelty and curiosity as determinants of exploratory behavior. British Journal of Psychology, 41, 68-80.

Besheer, J., \& Bevins, R. A. (2000a). Nicotine enhances acquisition of a $\mathrm{T}$-maze visual discrimination: Assessment of individual differences. Behavioural Pharmacology, 11, 613-620.

Besheer, J., \& Bevins, R. A. (2000b). The role of environmental familiarization in novel-object preference. Behavioural Processes, 50, 19-29.

Besheer, J., Jensen, H. C., \& Bevins, R. A. (1999). Dopamine antagonism in a novel-object recognition and a novel-object place conditioning preparation with rats. Behavioural Brain Research, 103, 35-44.

Besheer, J., Short, K. R., \& Bevins, R. A. (2001). Dopaminergic and cholinergic antagonism in a novel-object detection task with rats. Behavioural Brain Research, 126, 211-217.

Bevins, R. A. (2001). Novelty seeking and reward: Implications for the study of high-risk behaviors. Current Directions in Psychological Science, 10, 189-193.

Bevins, R. A., \& Bardo, M. T. (1999). Conditioned increase in place preference by access to novel objects: Antagonism by MK-801. Behavioural Brain Research, 99, 53-60.

Bevins, R. A., Besheer, J., Palmatier, M. I., Jensen, H. C., Pickett, K. S., \& Eurek, S. (2002). Novel-object place conditioning: Behavioral and dopaminergic processes in expression of novelty reward. Behavioural Brain Research, 129, 41-50.

Bevins, R. A., Besheer, J., \& Pickett, K. S. (2001). Nicotine-conditioned locomotor activity in rats: Dopaminergic and GABAergic influences on conditioned expression. Pharmacology Biochemistry and Behavior, 68, 135-145.

Bevins, R. A., Koznarova, J., \& Armiger, T. J. (2001). Environmental familiarization in rats: Differential effects of acute and chronic nicotine. Neurobiology of Learning and Memory, 75, 63-76.

Bozarth, M. A., Pudiak, C. M., \& KuoLee, R. (1998). Effect of chronic nicotine on brain stimulation reward: II. An escalating dose regimen. Behavioural Brain Research, 96, 189-194.

Carboni, E., Bortone, L., Giua, C., \& Di Chiara, G. (2000). Dissociation of physical abstinence signs from changes in extracellular dopamine in the nucleus accumbens and in the prefrontal cortex of nicotine dependent rats. Drug and Alcohol Dependence, 58, 93-102.

Carr, G. D., Fibiger, H. C., \& Phillips, A. G. (1989). Conditioned place preference as a measure $\sim$ f drug reward. In J. M. Liebman \& S. J. Cooper (Eds.), The neuropharmacological basis of reward (pp. 264319). New York: Oxford University Press. 
Centers for Disease Control and Prevention. (1994). Cigarette smoking among adults-United States, 1993. Morbidity and Mortality Weekly Report, 43, 925-929.

Centers for Disease Control and Prevention. (2001). Cigarette smoking among adults-United States, 1999. Morbidity and Mortality Weekly Report, 50, 869-873.

Cervo, L., \& Samanin, R. (1995). Effects of dopaminergic and glutamatergic receptor antagonists on the acquisition and expression of cocaine conditioning place preference. Brain Research, 673, 242-250.

Clarke, P. B., \& Kumar, R. (1983). The effects of nicotine on locomotor activity in nontolerant and tolerant rats. British Journal of Pharmacology, 78, 329-337.

Epping-Jordan, M. P., Watkins, S. S., Koob, G. F., \& Markou, A. (1998, May 7). Dramatic decreases in brain reward function during nicotine withdrawal. Nature, 393, 76-79.

Fung, Y. K., Schmid, M. I., Anderson, T. M., \& Lau, Y.-S. (1996). Effects of nicotine withdrawal on central dopaminergic systems. Pharmacology Biochemistry and Behavior, 53, 635-640.

Gianutosos, G., Drawbaugh, R., Hynes, M., \& Lal, H. (1975). The narcotic withdrawal syndrome in the rat. In S. Ehrenpreis \& A. Neidle (Eds.), Methods in narcotic research (pp. 293-309). New York: Marcel Dekker.

Harrison, A. A., Liem, Y. T. B., \& Markou, A. (2001). Fluoxetine combined with a serotonin-1 A receptor antagonist reversed reward deficits observed during nicotine and amphetamine withdrawal in rats. Neuropsychopharmacology, 25, 55-71.

Helton, D. R., Modlin, D. L., Tizzano, I. P., \& Rasmussen, K. (1993). Nicotine withdrawal: A behavioral assessment using schedule controlled responding, locomotor activity, and sensorimotor reactivity. Psychopharmacology (Berlin), 113, 205-210.

Hildebrand, B. E., Nomikos, G. G., Hertel, P., Schilström, B., \& Svensson, T. H. (1998). Reduced dopamine output in rats displaying a mecamylamine-precipitated nicotine withdrawal syndrome. Brain Research, 779, 214-225.

Hildebrand, B. E., Panagis, G., Svensson, T. H., \& Nomikos, G. G. (1999). Behavioral and biochemical manifestations of mecamylamine-precipitated nicotine withdrawal in the rat: Role of nicotinic receptors in the ventral tegmental area. Neuropsychopharmacology, 21, 560-574.

Hughes, J. R., Gust, S. W., Skoog, K., Keenan, R. M., \& Fenwick, I. W. (1991). Symptoms of tobacco withdrawal. Archives of General Psychiatry, 48, 52-59.

Hughes, J. R., Hatsukami, D. K., Pickens, R. W., Krahn, D., Malin, S., \& Luknic, A. (1984). Effect of nicotine on the tobacco withdrawal syndrome. Psychopharmacology (Berlin), 83, 82-87.

Irvine, E. E., Cheeta, S., \& File, S. E. (1999). Time-course of changes in the social interaction test of anxiety following acute and chronic administration of nicotine. Behavioural Pharmacology, 10, 691-697.

Killen, J. D., Fortmann, S. P., Newman, B., \& Varady, A. (1991). Prospective study of factors influencing the development of craving associated with smoking cessation. Psychopharmacology (Berlin), 105, 191-196.

Ksir, C., Hakan, R. L., \& Kellar K. I. (1987). Chronic nicotine and locomotor activity: Influences of exposure dose and test dose. Psychopharmacology (Berlin), 92, 25-29.

Levin, E. D., Lee, C., Rose, I. E., Reyes, A., Ellison, G., Jarvik, M., \& Gritz, E. (1990). Chronic nicotine and withdrawal effects on radial- arm maze performance in rats. Behavioral and Neural Biology, 53, 269-276.

Malin, D. H., Lake, J. R., Carter, V. A., Cunningham, J. S., Hebert, K. M., Conrad, D. L., \& Wilson, 0. B. (1994). The nicotinic antagonist mecamylamine precipitates nicotine abstinence syndrome in the rat. Psychopharmacology (Berlin), 115, 180-184.

Malin, D. H., Lake, J. R., Newlin-Maultsby, P., Roberts, L. K., Lanier, J. G., Carter, V. A., et al. (1992). Rodent model of nicotine abstinence syndrome. Pharmacology Biochemistry and Behavior, 43, 779-784.

Martin, B. R., Onaivi, E. S., \& Martin, T. J. (1989). What is the nature of mecamylamine's antagonism of the central effects of nicotine? Biochemical Pharmacology, 38, 3391-3397.

Mirza, N. R., \& Stolerman, I. P. (1998). Nicotine enhances sustained attention in the rat under specific task conditions. Psychopharmacology (Berlin), 138, 266-274.

Panagis, G., Hildebrand, B. E., Svensson, T. H., \& Nomikos, G. G. (2000). Selective $c$-fos induction and decreased dopamine release in the central nucleus of amygdala in rats displaying a mecamylamineprecipitated nicotine withdrawal syndrome. Synapse, 35, 15-25.

Sheldon, A. B. (1969). Preference for familiar versus novel stimuli as a function of the familiarity of the environment. Journal of Comparative and Physiological Psychology, 67, 516-521.

Shiffman, S., Johnston, A., Khayrallah, M., Elash, C. A., Gwaltney, C. J., Paty, J. A., et al. (2000). The effect of bupropion on nicotine craving and withdrawal. Psychopharmacology (Berlin), 148, 33-40.

Shippenberg, T. S., \& Herz, A. (1988). Motivational effects of opioids: Influence of D-1 versus D-2 receptor antagonists. European Journal of Pharmacology, 151, 233-242.

Stinus, L., Le Moal, M., \& Koob, G. F. (1990). Nucleus accumbens and amygdala are possible substrates for the aversive stimulus effects of opiate withdrawal. Neuroscience, 37, 767-773.

Sutton, M. A., \& Beninger, R. J. (1999). Psychopharmacology of conditioned reward: Evidence for a rewarding signal at D1-like dopamine receptors. Psychopharmacology (Berlin), 144, 95-110.

Suzuki, T., Ise, Y., Maeda, J., \& Misawa, M. (1999). Mecamylamine-precipitated nicotine-withdrawal aversion in Lewis and Fischer 344 inbred rat strains. European Journal of Pharmacology, 369, 159-162.

Suzuki, T., Ise, Y., Tsuda, M., Maeda, J., \& Misawa, M. (1996). Mecamylamine-precipitated nicotine-withdrawal aversion in rats. European Journal of Pharmacology, 314, 281-284.

Tzschentke, T. M. (1998). Measuring reward with the conditioned place preference paradigm: A comprehensive review of drug effects, recent progress and new issues. Progress in Neurobiology, 56, 613-672.

Watkins, S. S., Stinus, L., Koob, G. F., \& Markou, A. (2000). Reward and somatic changes during precipitated nicotine withdrawal in rats: Centrally and peripherally mediated effects. Journal of Pharmacology and Experimental Therapeutics, 292, 1053-1054.

West, R., \& Hajek, P. (1996). Randomised controlled trial of ondansetron in smoking cessation. Psychopharmacology (Berlin), 126, 95-96.
Submitted July 2002;

Revised September 2002;

Accepted October 2, 2002. 